\title{
THE KINEMATICAL MODEL OF THE ELBOW JOINT WITH ONE DEGREE OF FREEDOM
}

\author{
Petronela PARASCHIV ${ }^{1}$, Ramona CIMPOEȘU ${ }^{2 *}$, Mihai DUMITRU³, \\ Ciprian PARASCHIV ${ }^{4}$ \\ 1 “Gheorghe Asachi” Technical University, Sport Department, Iași, Romania \\ 2 "Gheorghe Asachi” Technical University, Materials Science and Engineering Faculty, Iași, Romania \\ 3 "Gheorghe Asachi" Technical University, Mechanics Faculty, Iași, Romania \\ 4 "Gr. T. Popa", University of Medicine and Pharmacy, Iași, Romania \\ *Corresponding author: ramonacimpoesu@gmail.com
}

DOI: $10.35189 /$ iphm.icpesk.2019.27

\begin{abstract}
From the kinematic point of view, the following parameters, constant or variable over time, can be highlighted in biomechanics: anthropometric dimensions, variable angular positions of the analysed movements, as well as the boundaries between which these angles vary. When considering the flexion-extension movement of the forearm to the arm, the structural model can be adopted as the simple pendulum. For such a structural model, the flexion-extension angle of the elbow joint is the generalised coordinates of the kinematic model. Since the purpose of the study is the post-traumatic recovery of the elbow joint, the appropriate kinematic model is a direct type, requiring some angular articulation variations of the elbow and determining the positions, speeds and accelerations of some points of interest the kinematic chain of the upper limb. Following the analysis of the movement of the structural model with a degree of freedom, the following conclusions can be highlighted: the passive flexion-extension movement of the forearm is oscillatory and can be compared to that of a simple pendulum; determining the period and frequency of oscillation for a mechanical system are necessary for the articular rehabilitation of the elbow; in the case of the first stages of joint rehabilitation, small angles of oscillation of the forearm can be fixed, with values smaller than 5 degrees.
\end{abstract}

Keywords: elbow joint, kinematic model, pendulum.

\section{Introduction}

From the kinematic point of view, the following parameters, constant or variable over time, can be highlighted in biomechanics: dimensional anthropometric (segmental dimensions, positions of given points, joint dimensions etc.); variable angular positions of the analysed movements, as well as the boundaries between which these angles vary; linear or angular positions of given points of interest; speeds and accelerations of points of interest (AbdelMalek, Yu, Yang, \& Nebel, 2004; Asada \& Fortier, 1999).

Depending on the link between these parameters, in biomechanics we can have: the direct kinematic analysis, where the dimensional anthropometric dimensions and the variable angular positions of the independent movements being analysed are known (these angular positions are called generalised coordinates of the structural model) and it is necessary to determine the variable positions of some given points, as well speeds and accelerations of those points corresponding to the analysed kinematic chain (Garland \& O'Hollaren, 1982; Jasińska-Choromańnska, 2006). This type of analysis can be applied in the case of the determination of motor, normal, pathological or performance parameters, post-traumatic recovery or recovery after an intense effort, starting from the anthropometric dimensions of the subject under consideration and from the angular values determined by rule, goniometer, of the independent movements studied (Paraschiv, Paraschiv, \& Cimpoeșu, 2015; Shiba et al., 1988; Tuohy, 2000).

Reverse kinematic analysis, where the variable positions of given points and/or the speeds and accelerations of those points are known, and the angular magnitudes of the independent movements analysed, as well as the anthropometric segment dimensions of the model structurally considered. This type of analysis can be applied with usefulness in performance sports. The mathematical general expression for the two types of analysis is (Paraschiv, Cimpoeșu, \& Paraschiv, 2015):

$X=f(\theta)$,

for direct kinematic analysis where $\theta$ is known and $\mathrm{X}$ must be determined and:

$\theta=\mathrm{f}^{-1}(\mathrm{X})$

for the reverse kinematic analysis, where $\mathrm{X}$ is known and $\theta$ is to be determined.

Given that the purpose of our study is the post-traumatic recovery of the elbow joint, the appropriate kinematic model is a direct type, requiring some angular articulation variations of the elbow and determining the positions, velocities and accelerations of some point's interest of the superior member's kinematic chain. 


\section{Topic Addressed}

\section{Numerical and experimental results}

When considering the flexion-extension movement of the forearm to the arm, the structural model can be adopted as the simple pendulum. For such a structural model (Figure 1), the flexion-extension angle of the elbow joint is the generalised coordinate (denoted by "q" below) of the kinematic model.
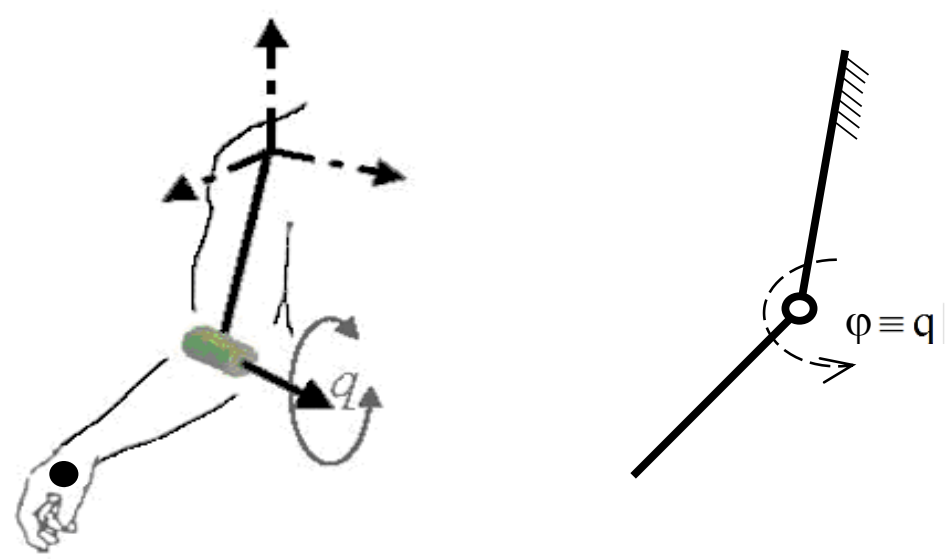

Figure 1. The joint model with a degree of freedom (Paraschiv, Cimpoeșu, \& Ursanu, 2014)

The position of an arbitrary point on the forearm using the polar coordinates can be written as follows:

$x=r \cdot \sin q$,

$\mathrm{y}=r \cdot \cos q$,

where: $r$ - is the length of the forearm or the length from the joint of the elbow to the point considered on the forearm; $\mathrm{q}-$ is the generalised coordinate, respectively the flexion-extension angle.

The " $\mathrm{r}$ " dimension being constant, the only variable describing the movement is the "q" angle made by the forearm with the vertical, as shown in Figure 2.

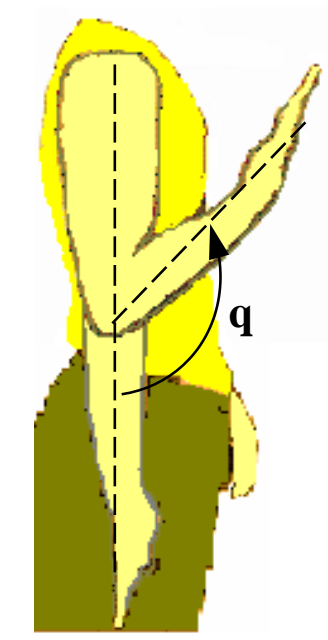

Figure 2. The pendulum movement of the forearm

The instantaneous movement of any point on the forearm measured along the circle arc is:

$\mathrm{ds}=\mathrm{r} \cdot \mathrm{dq}$,

where the instantaneous tangential speed results:

$\mathrm{v}_{\mathrm{t}}=\mathrm{r} \cdot \frac{\mathrm{dq}}{\mathrm{dt}}$

and instantaneous tangential acceleration of the shape: 
$a_{t}=r \cdot \frac{d^{2} q}{d t^{2}}$.

If the relationship (3) is derived in relation to time, the components of the velocity and acceleration of a given point on the forearm are obtained from an orthogonal coordinate axis system (denoted xOy and represented in Figure 3) of the form (Morrey, Chao, \& Hui, 1979; Hesse, Schmidt, Werner, \& Bardeleben, 2003):

$v_{x}=\dot{x}=r \cdot \frac{d q}{d t} \cdot \cos q=r \cdot \omega \cdot \cos q$,

$\mathrm{v}_{\mathrm{y}}=\dot{y}=-r \cdot \frac{d q}{d t} \cdot \sin q=-r \cdot \omega \cdot \sin q$,

and respectively:

$$
\begin{aligned}
& a_{x}=\ddot{x}=r \cdot \frac{d \omega}{d t} \cdot \cos q-r \cdot \omega \cdot \frac{d q}{d t} \cdot \sin q=r \cdot \varepsilon \cdot \cos q-r \cdot \omega^{2} \cdot \sin q, \\
& a_{y}=\ddot{y}=-r \cdot \frac{d \omega}{d t} \cdot \sin q-r \cdot \omega \cdot \frac{d q}{d t} \cdot \cos q=-r \cdot \varepsilon \cdot \sin q-r \cdot \omega^{2} \cdot \cos q,
\end{aligned}
$$

where $\omega$ represents the angular rotation speed of the forearm and $\varepsilon$ is the angular acceleration of the forearm.

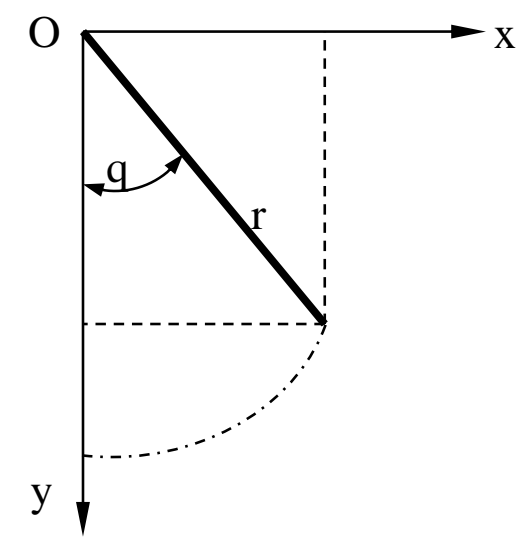

Figure 3. Orthogonal axis coordinate system

In order to plot the kinetic parameters variation graphs it is necessary first to know the distance " $r$ " from the elbow joint to the point on the forearm. For example, the point is the wrist, so the size $r$ is even the length of the forearm. This length, from the anthropometric data, is calculated with the relation:

$$
\mathrm{r}=1_{\mathrm{ab}}=0.146 \cdot \mathrm{H} \text {, }
$$

where $\mathrm{H}$ is the height of the analysed subject. For a subject with $\mathrm{H}=1.71[\mathrm{~m}]$, a forearm length of lab = $0.24966[\mathrm{~m}]$ results.

Figures 4 and 5 show the $\mathrm{x}$ and $\mathrm{y}$ coordinate variations of the "wrist' point on the forearm for the flexion movement between $0^{\circ}$ and $150^{\circ}$ of the elbow joint using the relationships (3).

In the case of velocity, it is necessary to know the angular velocity-extension velocity $\omega$, for which the variation graphs of components $\mathrm{v}_{\mathrm{x}}$ and $\mathrm{v}_{\mathrm{y}}$ were chosen, for which a constant value corresponding to the passive mobility during the post-traumatic recovery of the elbow was chosen. With $\omega=0.174$ [radians $/ \mathrm{s}$ ] and $\mathrm{r}=\mathrm{lab}=$ 0.24966 [m], Figures 6 and 7 represent the variation graphs of the velocity components in the forearm flexion movement using the analytical expressions (7). 
International Proceedings of Human Motricity/ ICPESK 2019

Supplementary Issue of Discobolul - Physical Education, Sport and Kinetotherapy Journal, 2019

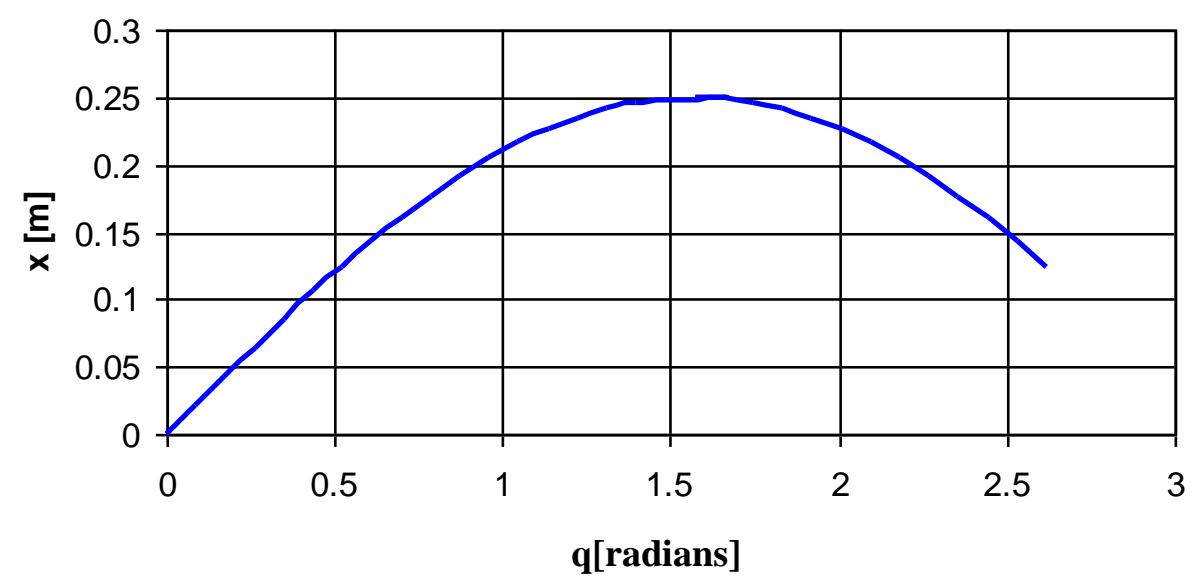

Figure 4. Variation of displacement $\mathrm{x}$ depends on the angle of flexion

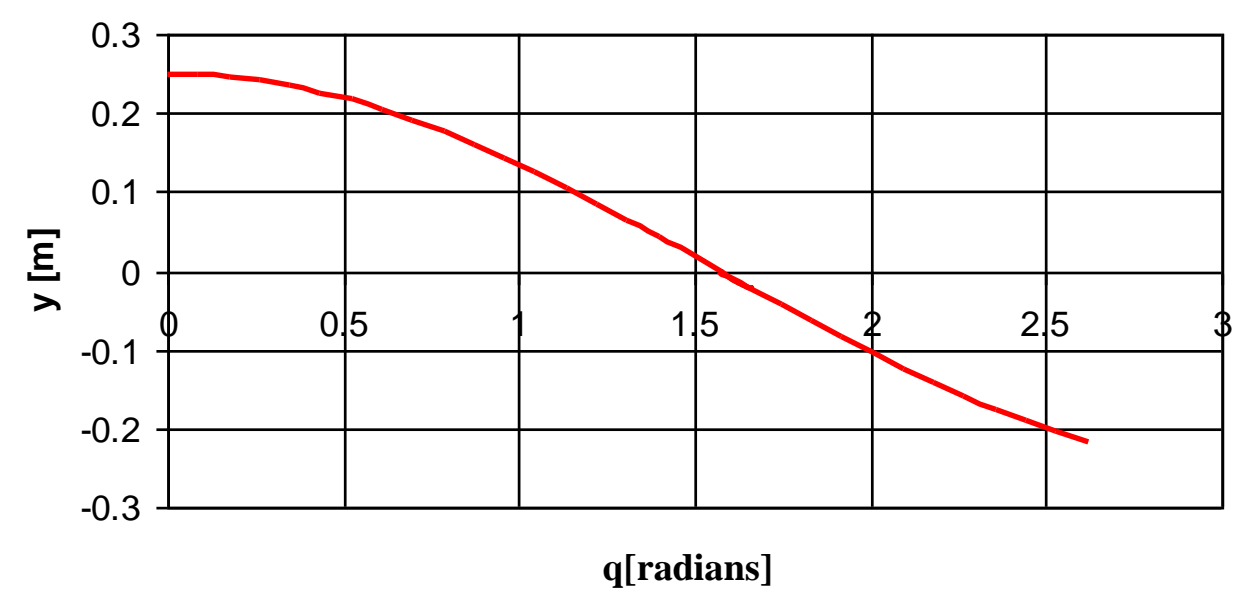

Figure 5. Variation of displacement y in relation to flexion angle

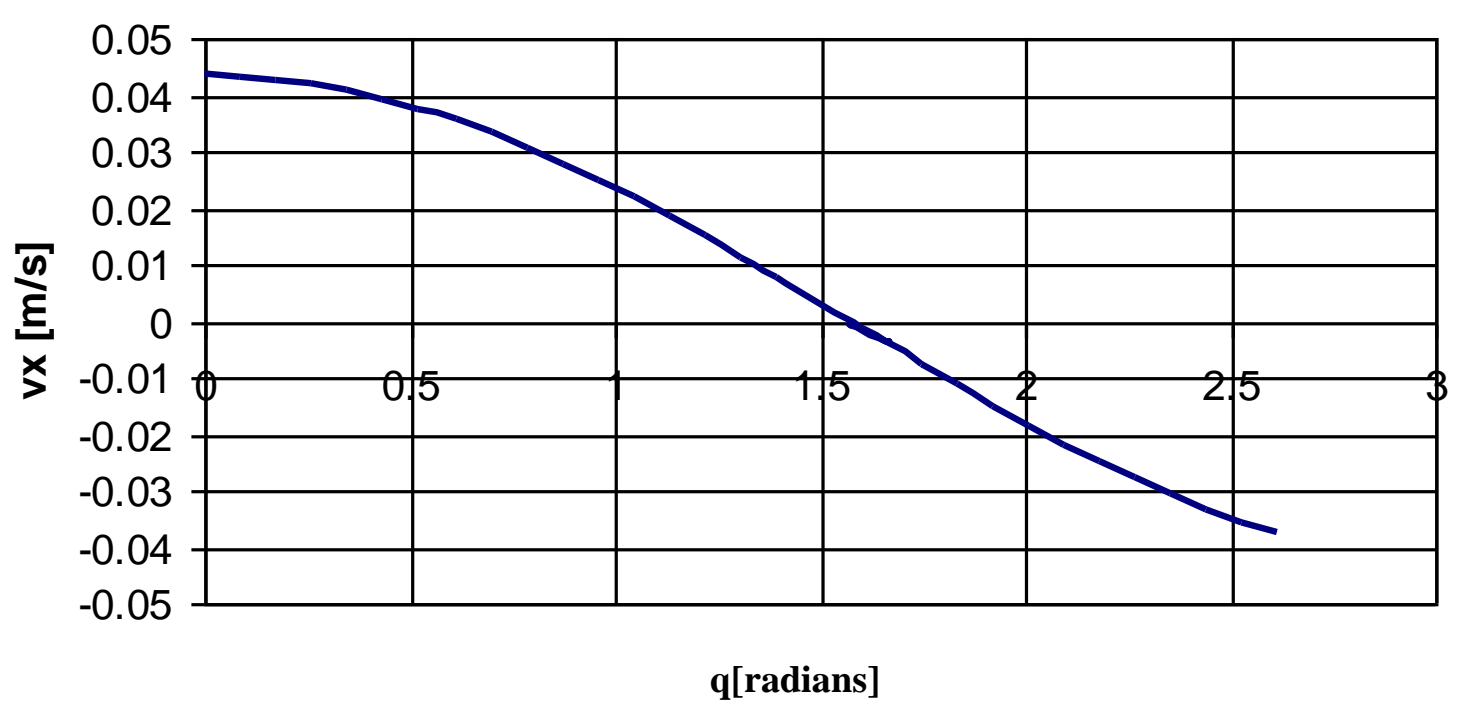

Figure 6. Velocity variation $\mathrm{v}_{\mathrm{x}}$ during flexion of the forearm 


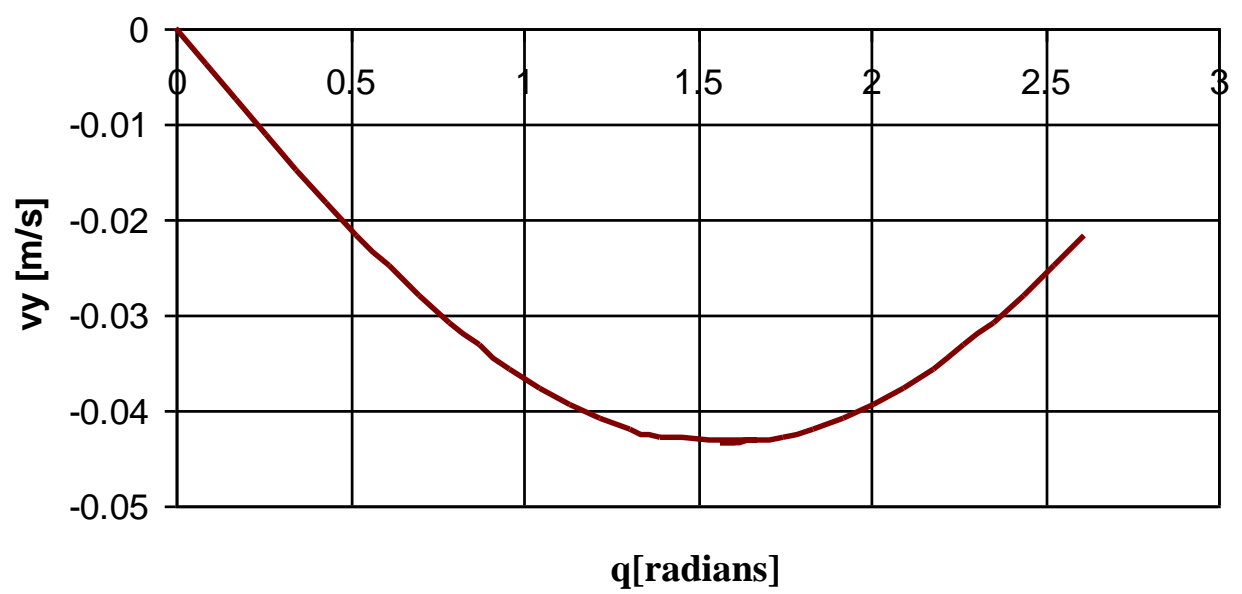

Figure 7. Variation of velocity $\mathrm{v}_{\mathrm{y}}$ during forearm flexion

The angular velocity $\omega$ allows the determination of the rotation period, knowing that:

$\omega=2 \cdot \pi / \mathrm{T}$,

the " $T$ " period resulting from the flexion-extension amplitude denoted $\varphi_{\max }$ as follows:

$\mathrm{T}=2 \cdot \pi \cdot \sqrt{\frac{\mathrm{r}}{\mathrm{g}}} \cdot\left(1+\frac{1}{4} \cdot \sin ^{2} \frac{\varphi_{\max }}{2}+\frac{9}{64} \cdot \sin ^{4} \frac{\varphi_{\max }}{2}+\ldots ..\right)$,

where: $\pi=3.14159265 \ldots$ and $g \approx 9.81\left[\mathrm{~m} / \mathrm{s}^{2}\right]-$ gravity acceleration, are constant values. Considering $\mathrm{r}=1_{\mathrm{ab}}=$ $0.24966[\mathrm{~m}]$ and $\varphi_{\max }=150^{\circ}$, results from mathematical calculations based on relations $(10)$ and $(11): \mathrm{T}=$ $1.358856[\mathrm{~s}]$ and $\omega=4.623878[\mathrm{rad} / \mathrm{s}]$.

The structural model of the simple pendulum can be easily determined on the basis of dynamic considerations, its equation of motion. Thus, from the expression of velocity, from relation (5): $\mathrm{v}=\mathrm{r} \cdot \dot{\mathrm{q}}=\mathrm{r} \cdot \omega$, it is possible to write the kinetic energy of the simple pendulum, of the form:

$\mathrm{E}_{\mathrm{c}}=\frac{1}{2} \cdot \mathrm{m} \cdot \mathrm{v}^{2}=\frac{1}{2} \cdot \mathrm{m} \cdot \mathrm{r}^{2} \cdot \dot{\mathrm{q}}^{2}$,

where " $\mathrm{m}$ " represents, in the analysed case, the mass of the forearm.

The potential energy of the pendulum is:

$\mathrm{E}_{\mathrm{p}}=\mathrm{m} \cdot \mathrm{g} \cdot(\mathrm{r}-\mathrm{r} \cdot \cos \mathrm{q})$,

where $g=9.81\left[\mathrm{~m} / \mathrm{s}^{2}\right]$ represents the acceleration of gravity.

The pendulum motion equation can be simply determined from the Lagrange equations of the second type, of the form:

$\frac{\mathrm{d}}{\mathrm{dt}}\left(\frac{\partial \mathrm{L}}{\partial \dot{\mathrm{q}}}\right)-\frac{\partial \mathrm{L}}{\partial \mathrm{q}}=0$

where: $L=E_{c}-E_{p}=\frac{1}{2} \cdot m \cdot r^{2} \cdot \dot{q}^{2}-m \cdot g \cdot(\mathrm{r}-r \cdot \cos q)$ is Lagrange's function, either from the system's total energy conservation theorem, of the form:

$\frac{\mathrm{dE}}{\mathrm{dt}}=0$,

where: $\mathrm{E}=\mathrm{E}_{\mathrm{c}}+\mathrm{E}_{\mathrm{p}}=\frac{1}{2} \cdot \mathrm{m} \cdot \mathrm{r}^{2} \cdot \dot{\mathrm{q}}^{2}+\mathrm{m} \cdot \mathrm{g} \cdot(\mathrm{r}-\mathrm{r} \cdot \cos \mathrm{q})$ represent the total energy.

Using the theory of total energy conservation, we obtain the general equation of the pendulum movement, of the form:

$\mathrm{m} \cdot \mathrm{r}^{2} \cdot \dot{\mathrm{q}} \cdot \ddot{\mathrm{q}}+\mathrm{m} \cdot \mathrm{g} \cdot \mathrm{r} \cdot \dot{\mathrm{q}} \cdot \sin \mathrm{q}=0$,

where, by taking into account the common factor, the expression:

$\mathrm{m} \cdot \mathrm{r} \cdot \dot{\mathrm{q}} \cdot(\mathrm{r} \cdot \ddot{\mathrm{q}}+\mathrm{g} \cdot \sin \mathrm{q})=0$.

Equation (17) leads to two solutions, namely:

from $\dot{\mathrm{q}}=0$, it results that the pendulum has no movement, being at rest; 
- from $\ddot{\mathrm{q}}+\frac{\mathrm{g}}{\mathrm{r}} \cdot \sin \mathrm{q}=0$, there are several discussions, namely:

- if the total energy $\mathrm{E}$ is less than a certain critical value, then the pendulum will move periodically, back and forth, which means a flexion-extension movement of the forearm;

- if the energy $\mathrm{E}$ is greater than the critical value, then the pendulum rotates around the point of suspension, which means that the movement of the forearm tends to damage the articulation of the elbow destructively;

- if energy $\mathrm{E}$ is equal to critical value then there are two possibilities: first, if the pendulum starts the motion, then it will approach the vertical position more and more but without touching it in a finite time; the second is the situation where the pendulum stands exactly in the upright position, where there is an undefined time.

The critical energy value $E$ is the value of the potential energy for which $q= \pm \pi$, this leading to the expression: $\mathrm{E}_{\mathrm{cr}}=2 \cdot \mathrm{m} \cdot \mathrm{g} \cdot \mathrm{r}$.

Using the expression of critical energy, the law of variation of the generalised coordinate can be determined $q$ $=\mathrm{q}(\mathrm{t})$, of the Energy Conservation Act:

$2 \cdot \mathrm{m} \cdot \mathrm{g} \cdot \mathrm{r}=\frac{1}{2} \cdot \mathrm{m} \cdot \mathrm{r}^{2} \cdot \dot{\mathrm{q}}^{2}+\mathrm{m} \cdot \mathrm{g} \cdot(\mathrm{r}-\mathrm{r} \cdot \cos \mathrm{q})$,

which is rearranged as:

$\dot{\mathrm{q}}=2 \cdot \omega_{0} \cdot \cos \frac{\mathrm{q}}{2}$,

where $\omega_{0}=\sqrt{\frac{\mathrm{g}}{\mathrm{r}}}$ is the small oscillations frequency.

Considering that the pendulum starts the movement from $\mathrm{q}=0$, the solution of equation (19) is:

$\mathrm{q}(\mathrm{t})=2 \cdot \arcsin \left(\frac{1-\mathrm{e}^{-2 \omega_{0} \cdot \mathrm{t}}}{1+\mathrm{e}^{-2 \omega_{0} \cdot \mathrm{t}}}\right)$,

which shows that when the time " $\mathrm{t}$ " increases, the value of " $\mathrm{q}$ " tends towards $\pi$, the situation not being encountered in the case of the movement of the forearm, knowing that the flexion cannot exceed $150^{\circ} \ldots .160^{\circ}$.

Equation $\ddot{\mathrm{q}}+\frac{\mathrm{g}}{\mathrm{r}} \cdot \sin \mathrm{q}=0$ can be re-write under this form:

$\frac{\mathrm{d}}{\mathrm{dt}}\left(\frac{1}{2} \cdot \dot{\mathrm{q}}^{2}\right)-\frac{\mathrm{g}}{\mathrm{r}} \cdot \frac{\mathrm{d}}{\mathrm{dt}}(\cos \mathrm{q})=0, \mathrm{t} \geq \mathrm{t}_{0}$.

Equation (21) can be integrated with time to obtain the expression:

$[\dot{\mathrm{q}}(\mathrm{t})]^{2}-\left[\dot{\mathrm{q}}\left(\mathrm{t}_{0}\right)\right]^{2}=2 \cdot \frac{\mathrm{g}}{\mathrm{r}} \cdot\left[\cos \mathrm{q}(\mathrm{t})-\cos \mathrm{q}\left(\mathrm{t}_{0}\right)\right]$,

where: $\mathrm{q}\left(\mathrm{t}_{0}\right)=\mathrm{q}_{0}$ represents the initial position of the pendulum, respectively the initial position of the forearm in its flexion-extension movement; this value can be $\mathrm{q}=0$.

Taking into account the relation (22) and the fact that the tangential velocity is $\mathrm{v}=\mathrm{r} \cdot \dot{\mathrm{q}}$ the following expression is found:

$\mathrm{v}^{2}=2 \cdot \mathrm{g} \cdot \mathrm{r} \cdot\left(\cos \mathrm{q}-\cos \mathrm{q}_{0}\right)$,

considering the initial speed $\mathrm{v}_{0}=\mathrm{r} \cdot \dot{\mathrm{q}}\left(\mathrm{t}_{0}\right)=0$.

From relation (23) results:

$\mathrm{r} \cdot \dot{\mathrm{q}}=\sqrt{2 \cdot \mathrm{g} \cdot \mathrm{r} \cdot\left(\cos \mathrm{q}-\cos \mathrm{q}_{0}\right)}$,

from where:

$\frac{\mathrm{dq}}{\mathrm{dt}}=\sqrt{\frac{4 \mathrm{~g}}{\mathrm{r}} \cdot\left(\sin ^{2} \frac{\mathrm{q}_{0}}{2}-\sin ^{2} \frac{\mathrm{q}}{2}\right)}$.

By separating the variables and integrating both members of the relationship (25), between the flexionextension extension limits of the forearm, the expression of the pendulum rotation period is expressed as:

$$
\mathrm{T}=\sqrt{\frac{\mathrm{r}}{\mathrm{g}}} \cdot \int_{0}^{150^{\circ}} \frac{\mathrm{dq}}{\sqrt{\sin ^{2} \frac{\mathrm{q}_{0}}{2}-\sin ^{2} \frac{\mathrm{q}}{2}}},
$$


a relation that is numerically equivalent to the expression (11) for which a value has previously been calculated.

\section{Conclusion}

Following the analysis of the movement of the structural model with a degree of freedom, the following conclusions can be highlighted:

- the passive flexion-extension movement of the forearm is oscillatory and can be compared to that of a simple pendulum;

- the period and frequency of oscillation, calculated with mathematical relationships such as (10), (11), (26), are values necessary for a mechanical system of joint elbow rehabilitation, a system by which the simple pendulum motion is imitated, controlled by the forearm;

- in the case of the first stages of articular rehabilitation, small angles of oscillation of the forearm and angles $\leq 5^{\circ}$ can be fixed so that the solution of the motion equation is $q=q_{m} \cdot \sin \omega t$, where $q_{m}$ is the angular amplitude (which is fixed), $\omega=2 \pi / \mathrm{T}$ is pulsation and $\mathrm{T}=2 \pi \cdot \sqrt{\frac{\mathrm{r}}{\mathrm{g}}}$ the period of angular oscillations.

\section{References}

Abdel-Malek, K., Yu, W., Yang, J., \& Nebel, K. (2004). A mathematical method for ergonomic-based design: Placement. International Journal of Industrial Ergonomics, 34(5), 375-394. https://doi.org/10.1016/j.ergon.2004.05.001

Asada, H. H., \& Fortier, J. (1999). Using an instrument-glove and a biomechanical model for human machine coordination and task recognition. Progress Report, 2-4, 1-16.

Garland, D. E., \& O'Hollaren, R. M. (1982). Fractures and dislocations about the elbow in the head-injured adult. Clinical Orthopaedics, 168, 38-41. [In Polish]

Hesse, S., Schmidt, H., Werner, C., \& Bardeleben, A. (2003). Upper and lower extremity robotic devices for rehabilitation and for studying motor control. Current Opinion in Neurology, 16(6), 705-710. https://doi.org/10.1097/01.wco.0000102630.16692.38

Jasińska-Choromańska, D. (2006). Diagnosis of bone fracture healing using external fixators. Paper presented at the International Conference "Biomechanics 2006", Zakopane.

Morrey, B. F., Chao, E. Y. S., \& Hui, F. C. (1979). Biomechanical study of the elbow following excision of the radial head. The Journal of Bone and Joint Surgery, 61(1), 63-68. Retrieved from https://www.ncbi.nlm.nih.gov/pubmed/759438

Paraschiv, P., Cimpoeșu, N., \& Ursanu, A. (2014). Theoretical and experimental determination of the muscle strength for the kinetotherapy rehabilitation of the elbow joint after an immobilization period. Procedia Social and Behavioral Sciences, 117, 539-546. https://doi.org/10.1016/j.sbspro.2014.02.259

Paraschiv, C., Paraschiv, P., \& Cimpoeșu, R. (2015). Usage of two-freedom degrees for modelling of a complex wrist. In 4th International Congress of Physical Education, Sport and Kinetotherapy (ICPESK 2014, pp. 363369). Bologna: Medimond.

Paraschiv, P., Cimpoeșu, N., \& Paraschiv, C. (2015). Radius deformations and stress appearance at external solicitations. In 4th International Congress of Physical Education, Sport and Kinetotherapy (ICPESK 2014, pp. 375-381). Bologna: Medimond.

Shiba, R., Sorbie, C., Siu, D. W., Bryant, J. T., Cooke, T. D. V., \& Wevers, H. W. (1988). Geometry of the elbow joint. Journal of Orthopaedic Research, 6, 897-906.

Tuohy, B. T. (2000). Development of a biodynamic module for forensic applications (Master of Science in Engineering Mechanics). Retrieved from https://vtechworks.lib.vt.edu/bitstream/handle/10919/35940/BrentETD.pdf?sequence=1\&isAllowed=y 